\title{
CEREBRAL ISCHAEMIA AND EXTRA-CEREBRAL VASCULAR DISEASE
}

\author{
Edward C. Hutchinson, M.D., M.R.C.P. \\ Consulting Neurologist, Midland Centre for Neuro-Surgery and Stoke-on-Trent \\ Peter O. YATes, M.D. \\ Senior Lecturer in Neuro-Pathology, University of Manchester
}

At a time when the problems of degenerative disease and ageing are claiming the attention of both the clinician and the pathologist it is not surprising that interest in cerebral vascular disease has been re-awakened. Lack of effective treatment in the past encouraged no great interest in defining critically the details of the various clinical pictures and the corresponding variations in the underlying pathological lesions. It is only within the last 15 years that Aring (1945) and Hicks and Warren (195.I) found it necessary to put forward vasospasm as a possible cause of cerebral ischaemia in many of their cases of cerebral infarction, since they were unable to demonstrate any adequate obstructive lesion of the appropriate intracranial vessel. The difficulties of accepting vasospasm as a mechanism of cerebral infarction have been summarised by Pickering (1959). Moreover, it is now realised that disease of the carotid and vertebral arteries in their extracranial course is responsible for a proportion of previously unexplained cases of cerebral ischaemia encountered by both the physician and the pathologist.

This review is concerned with the recent advances in the aetiology, the clinical presentations and the treatment of patients with extracranial cerebral vascular disease; the term 'extracranial vascular disease' is used to emphasise the important pathological fact that in these cases it is not unusual for more than one of the major vessels' in the neck to be affected by disease. An appreciation of the importance of extracranial arterial disease, together with advances in vascular surgery, anaesthesia and radiology, has encouraged the successful surgical attack upon these arterial lesions-a development which is the most important recent advance in the treatment of cerebral vascular disease.

\section{Pathology}

In the main the pathological changes in the vessels in occlusive cerebral vascular disease are $\stackrel{3}{\omega}$. those of athero-sclerosis although giant cell arteritis, polyarteritis nodosa, thrombo-angiitis obliterans, and the rare 'pulseless disease,' have all been described. The clinical and pathological

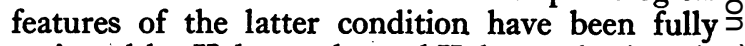
reviewed by Kalmansohn and Kalmansohn (1957).

It is an accepted pathological observation that the pattern of vessel involvement in atheroma varies considerably from individual to individualthe pattern being determined by the size of the $\overrightarrow{0}$ vessel involved. In some cases the small, in other the large, vessels are predominantly affected. Sucf a distinction is frequently seen in cerebral vascular disease where the smaller intracranial arteries may be free of disease when the changes are far advanced in the larger extracranial arteries. Indeed it is $\stackrel{2}{\varnothing}$ important in any study of cerebral ischaemia to $\unrhd$ appreciate that occlusive disease of the carotid $\overrightarrow{0}$ artery occurs as frequently as occlusion of the middle cerebral artery, and that occlusion of the vertebral artery occurs more frequently in its cervical course than does occlusion of either the basilar or the posterior inferior cerebellar artery. A point of additional significance in the pathology 3 . of these cases-important to the surgeon-is the frequent localization of atheromatous disease in the $\frac{1}{3}$ internal carotid artery at a point $\mathbf{I ~} \mathrm{cm}$. above its origin and in the vertebral artery to the portion? lying between its origin from the subclavian artery $\frac{D}{0}$ and the vertebral arterial canal. Whatever the fundamental pathological lesion may be in $N$ atheroma haemorrhage into the base of the $N$ plaques in the carotid artery frequently occurs $N$ (Yates, 1954) and Rob (1959) has emphasized the importance of such an event in the pathogenesis of clinical symptoms in carotid arterial occlusion.

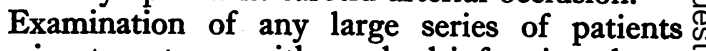
coming to autopsy with cerebral infarction shows ? that between one-third and one-half of these $\overline{0}$ patients have advanced disease of the carotid and $\frac{}{\triangleright}$ 
the vertebral arteries in various combinations. Commonly, arterial disease is usually found in both the carotid and the vertebral arteries producing in the four vessels varying degrees of stenosis and occlusion. Less frequently the disease is confined to the carotid artery alone and, least common of all, the atheromatous changes are present only in the vertebral arteries.

It is now generally agreed that the development of symptoms in cerebral vascular disease may be related in part to the available collateral circulation. The important sites in the development of a collateral circulation in cerebral vascular disease are as follows:-

I. Anastomoses over the cerebral hemispheres and the cerebellum between the superficial branches of the major arteries-points where considerable anastomotic channels have been demonstrated by injection studies (Van der Eecken and Adams, I953). The importance of these anastomoses in occlusion of the middle cerebral artery has already been demonstrated in life by angiography (Rosegay and Welch, 1954).

2. Anastomoses between the carotid and the vertebral arteries when one or other is occluded, and the most important site of anastomosis under these circumstances is the Circle of Willis.

3. The potential collateral circulations between the external and internal carotid arteries via the ophthalmic arteries; the anatomy of these has been fully defined by Weiss (1938).

4. When considering the collateral circulation in any given case the effect of congenital vascular abnormalities must be considered. Anomalies of the Circle of Willis, unimportant in health, may be vital in disease if the anomaly affects the establishment of an effective collateral circulation through the Circle of Willis when the carotid, vertebral or intracerebral arteries are occluded. Similarly, variation in size of the vertebral artery, present to a significant degree in three-quarters of the patients coming to post mortem (Stopford, I92I) may assume great importance if the larger of the two is occluded by disease.

Areas of cerebral infarction in the brains of patients dying from ischaemic cerebral vascular disease are frequently multiple, and may be present in both the cerebellum and the cerebral hemispheres if both the carotid and the vertebral arteries are involved by disease. The infarct may be found within the territory of an intracerebral artery if the atheroma is confined to the local vessel of supply but the infarct may also occur at junctional territories-for example the junction of the middle and posterior cerebral arteries over the posterior parietal areas-if the cause of infarction is occlusion of the carotid and the vertebral arteries which has led to a reduction of the total cerebral blood flow.

\section{Clinical Aspects}

There is already an extensive body of literature on the neurological findings in cases of internal carotid thrombosis and a detailed review has already appeared in this journal (Behrman, 1954); all the available literature emphasises the wide variations in the clinical presentation in these cases. These variations are in part determined by the degree and distribution of the atheroma in the extracranial vessels so that the diagnosis of internal carotid thrombosis applied to every case may obscure the fact that the symptoms may also be due to extensive disease of the other major vessels of supply to the brain.

Surgical experience has shown that ligation of one carotid artery may be carried out in the younger patient without sequelae, and it is not surprising, therefore, that occlusion of one carotid artery by atheroma may be present without symptoms. One of the most common presentations of internal carotid thrombosis is for the patient to present with symptoms and signs almost identical with those of middle cerebral thrombosis. The usual sequence of events in these patients is for the thrombus to extend from the site of disease at the origin of the carotid artery across the Circle of Willis and thus occlude the middle cerebral and frequently the anterior cerebral artery. Clinical difficulties may occur in these circumstances when the infarcted brain becomes so oedematous as to produce signs of raised intracranial pressure (Clarke and Harris, 1958).

Extension of the thrombus from the internal carotid artery directly into the intracerebral arteries has been observed, not only in atheromatous disease of the carotid artery, but also as a complication of pregnancy in the postpartum period.

\section{Case I.}

The patient, a woman of 24 years of age, had been delivered normally of a child ten days before admission to hospital. A week after delivery the patient complained of a sudden attack of involuntary movements of the right hand, and it was probable that during this first attack, she was dysphasic. The attack lasted for some 20 minutes, and when she recovered she complained of headache over the left eye. Following this the patient was depressed and confused, and it was clear that from time to time she lost her speech for periods of several hours on each occasion. The following day she was transferred to hospital and she was seen for the first time I I days after delivery. At this time the patient was semi-conscious and totally 
aphasic. There was a dense right hemiplegia with a marked degree of sensory loss, but the modalities affected could not be assessed in view of the patient's mental state. The patient was of slight build and the neck was slim, so that examination of the cervical vessels could be carried out with ease. Normal pulsation of the carotid artery on the right side could be felt, but on the left side there was no carotid pulsation, and in addition a hard and thickened cord was present. The cerebro-spinal fluid showed a resting pressure of $190 \mathrm{~mm}$. of water, but the fluid was clear and colourless and was of normal cellular and protein content. The patient died in the early hours of the following morning, 12 days after delivery.

At post mortem the essential findings concerned the central nervous system and the carotid arteries. There was a complete thrombosis of the left common carotid artery from its point of origin in the mediastinum extending into the middle cerebral artery within the skull. The left cerebral hemisphere was infarcted and oedematous. There were no other significant features in the general post mortem.

The clinical presentation which has received the most attention in the literature is that in which transient episodes of neurological disturbance are a feature of the history. The patient may be symptom free between these episodes or they may occur against a background of a progressive neurological deficit. Disturbances of speech, motor power and sensation, may all occur, as may transient visual disturbances. These last are usually represented by attacks of monocular blindness affecting usually the same side as that of the diseased carotid artery. The attacks of disturbance of vision may last for minutes or several hours, and they may disappear completely after a time or permanent impairment of vision may develop as a result of retinal artery occlusion.

These visual disturbances associated with carotid artery stenosis have led to the measurement of retinal artery blood pressure with an ophthalmodynamometer. The results, in what may be a promising investigation in this condition, have been reviewed by Hoyt (1959).

In this group of patients with recurring clinical episodes it is not uncommon at post mortem to find atheroma causing stenosis in more than one of the major arteries, and it seems reasonable to suppose that many of these clinical episodes in this group represent transient failures of the collateral circulation-a circulation which may depend either on the contralateral carotid artery or on the vertebral arteries. Sudden failure of the cerebral circulation as a whole may occur in these cases by the sudden reduction of the cerebral blood flow by the elevation of an atheromatous plaque in the carotid artery by haemorrhage into the depths of the plaque as described by Rob (r959). The following patient is an illustration of the clinical history and pathological findings in this group of cases where the atheroma involves both the carotid? and the vertebral arteries:-

\section{Case 2}

The patient, a male aged 5 I years, complainec $\frac{\Phi}{8}$ that one year before admission he had suddenlyes developed weakness of the right hand and, equally $\overrightarrow{0}$ suddenly, lost the sight of his right eye. Thesesigns cleared completely, but six months later he् noted a further transient episode of weakness of the right arm and leg with some slurring of speech 5 A month after this he developed weakness of the left arm, leg and face with a return of the speecho disturbance. When admitted to hospital the abnormalities in the nervous system comprised $\vec{a}$ left homonymous hemianopia with sensory im pairment down the left side of the body. There was also clear evidence on neurological examina- tion of a parietal lobe lesion.

A right carotid angiogram showed completêे occlusion of the internal carotid artery. His sub sequent progress was one of slow deterioratign $\overrightarrow{0}$ with occasional episodes of weakness of both ti right and the left sides of the body. For the lit two months of his life the patient was confusedo and agitated, sinking into coma in the last week of his life.

At post mortem the significant abnormalitieso

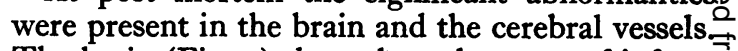
The brain (Fig. I) showed patchy areas of infarc-oํㅡㅇ tion throughout both the anterior cerebral and middle cerebral arterial territories. These changes were estimated to be of two to three months: duration, and were most marked in the right hemisphere, but both the brain stem and basal ganglias. were free of infarction. In the cerebellum there? was a recent bilateral infarction involving almosti the whole of the territory of the superior cerebellar artery.

On examining the vessels the intracranial vessels showed minimal atheroma only. In the extracranial portion (Fig. 2) both internal carotid arteries showed old, shrunken, re-organized thrombus extending on both sides from the neck ${ }_{0}$ beyond the origin of the ophthalmic arteries, butew not into the intracranial vessels. In the sinuso region overlying the old organized thrombus fairlyo recent new thrombus had been deposited. The left vertebral artery showed an old organized? thrombus from its origin to the level of the secondo cervical vertebra. The right vertebral artery $\overrightarrow{\vec{D}}$ showed minor atheromatous plaques only. 

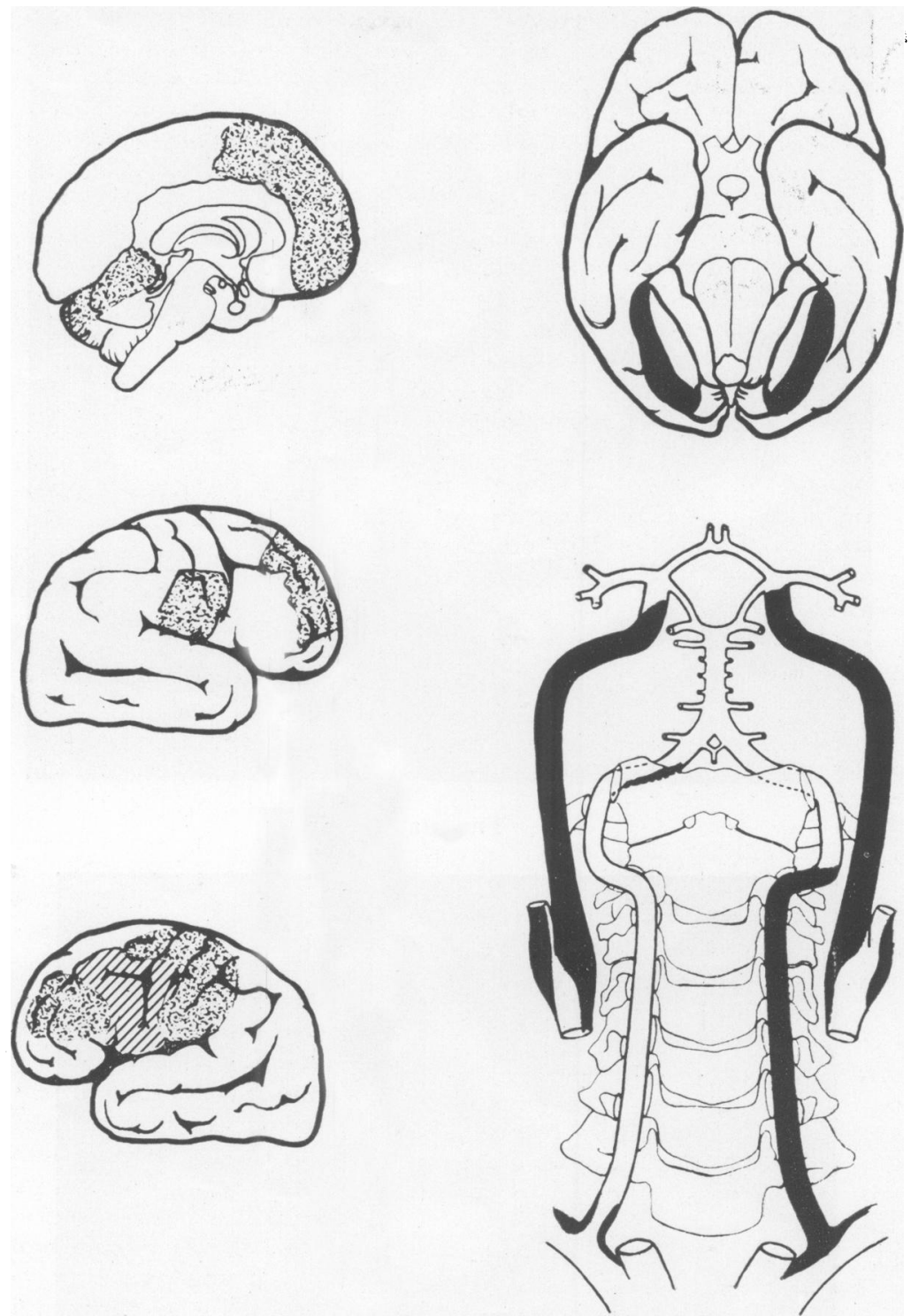

FIG. I.-Diagrams showing distribution of cerebral and cerebellar infarcts and the extent of occlusion of the major arteries supplying the brain.

The clinical history in these patients may very well suggest a vascular lesion as the underlying cause, but sometimes the clinical diagnosis may not be by any means so easy. Dementia may dominate the clinical picture from beginning to end as Fisher (1954) has already emphasized. Moreover the clinical history may be one of a slow and steadily progressive picture of disease of one or both cerebral hemispheres so that the clinical presentation is indistinguishable without angiography from an intracranial neoplasm.

Carotid and vertebral arterial disease may be present without symptoms, but any illness producing sudden hypotension may unmask the inadequacy of the cerebral circulation. Haematemesis, coronary occlusion, post-operative shock, may all produce this effect, as may any other cause of hypotension. Loss of consciousness or death may follow the episode of hypotension, or, if the patient survives, transient or permanent neurological signs may be apparent.

Disease of the vertebral arteries in their cervical course may be the sole manifestation of cerebral vascular disease. Resulting ischaemia may give 


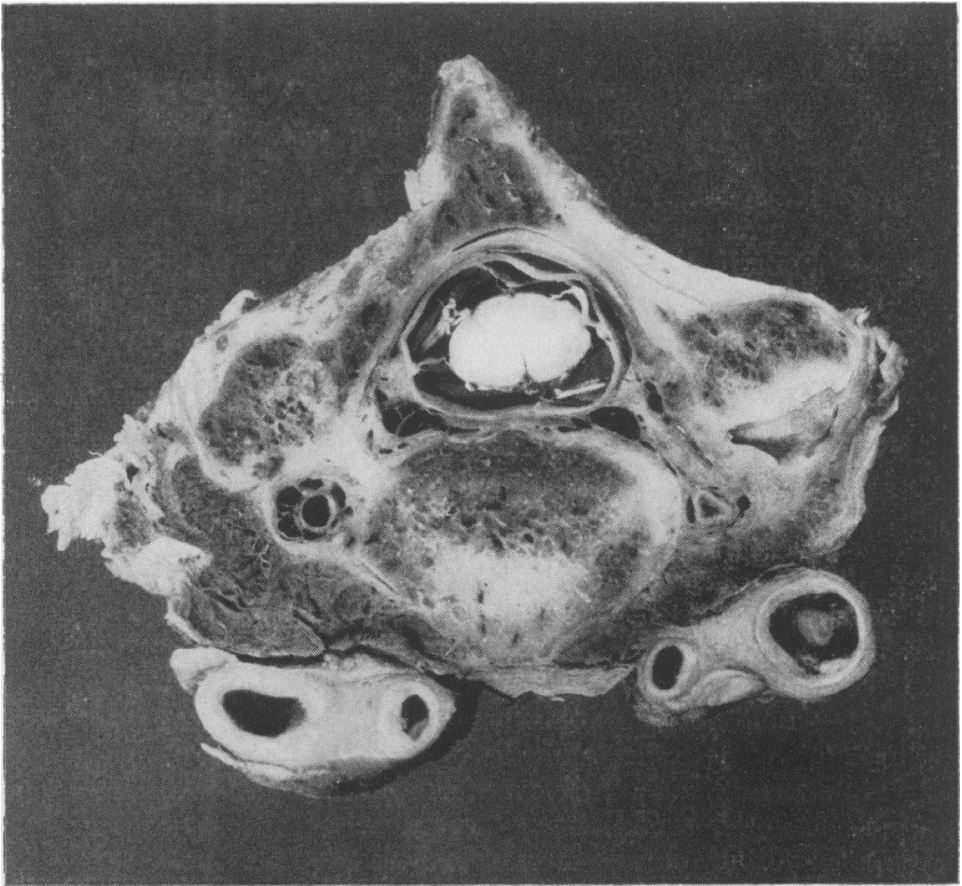

FiG. 2(a).

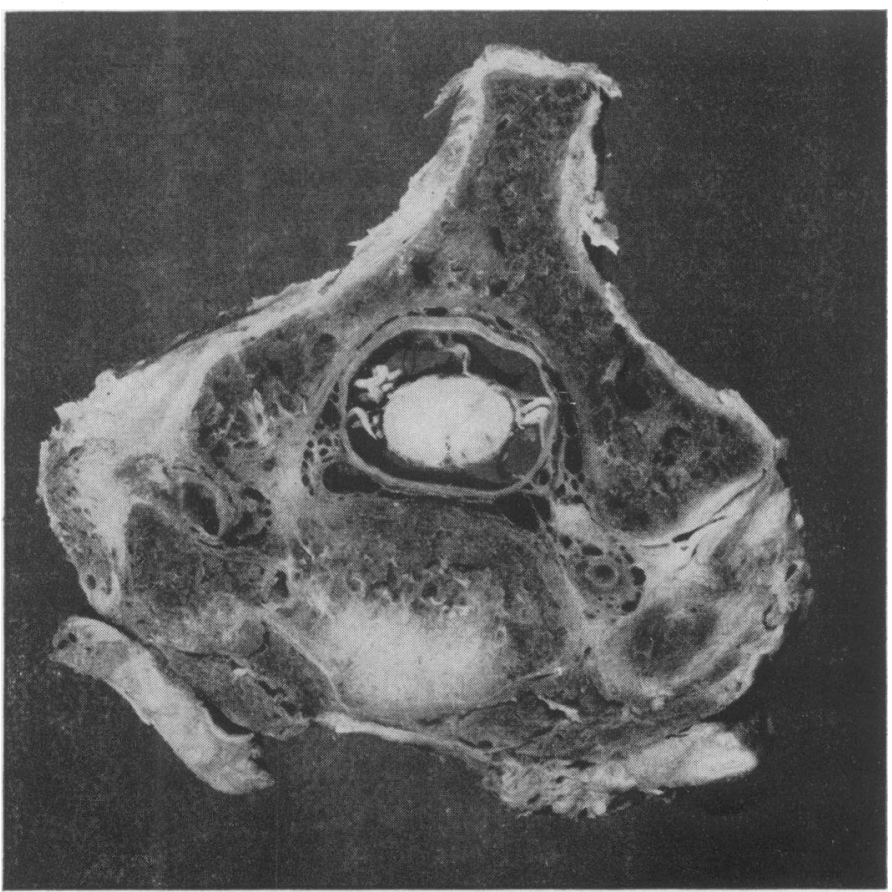

Fig. 2(b).

Fig. 2.-Horizontal sections of the neck to show the state of the four major cerebral vessels (a) just above the carotid bifurcation to show both internal carotids occluded by thrombus and the left vertebral by organized thrombus, $(b)$ to show both internal carotid arteries shrunken and fused with surrounding tissue at level of third cervical vertebra. 
rise to symptoms indicative of a brain stem, cerebellar, or occipital lobe infarction (Hutchinson and Yates, 1957; Crawford et al., 1958; Duffy and Grant, 1958). The clinical syndrome resulting from disease of the vertebral artery in its cervical course bears a close relationship to the syndrome of basilar artery occlusion as it was defined by Kubik and Adams (1946) and later by Millikan and Seikert (1955), a similarity which is not surprising since both ultimately supply approximately the same territory of the brain. Many points of the syndrome remain to be classified, one of the most important being the natural history of the disease. As far as personal experience and published work will allow the patients may be divided into two groups, the division depending upon whether or not transient neurological symptoms indicative of brain stem ischaemia occur before the final fatal illness.

If no such transient neurological episodes occur and the patient when seen by the physican, for the first time, is in coma-a not infrequent occurrence in these patients - then vertebral artery occlusion cannot be distinguished from any other form of cerebral vascular disease. However, the loss of consciousness may be slow and if seen in the early stages the signs of brain stem ischaemia may be elicited and the possibility of vertebral arterial occlusion may be considered, under these circumstances. The diagnosis may be very much easier if transient neurological episodes occur before the final fatal illness. One may anticipate from the fact that the symptoms arise in the brain stem that the symptomatology may be very variable. Attacks of double vision, vertigo, unsteadiness and incoordination of the limbs and mental confusion, have all been described. Visual disturbances too, are frequent and this is not surprising, since occipital lobe infarction has been noted in these patients at post mortem as a result of vertebral arterial occlusion. Crawford et al. (1959) mentioned bilateral blindness and hemianopia as occurring in their cases of vertebral arterial occlusion, and it may well be, as Symonds and Mackenzie (1957) have suggested, that some examples of the syndrome of the bilateral posterior cerebral artery occlusion may be due to disease of the vertebral arteries in their cervical course. The following clinical account gives the details of a patient who complained of transient neurological episodes prior to the final fatal episode.

\section{Case 3}

The patient, a man of 57 years, was operated on in 1945 for a mastoid infection and following operation remained well for 12 years. In 1958 he first complained of vertigo and vomiting. In spite of the previous medical history no adequate aural cause could be found for these attacks, and they continued at frequent intervals until June of 1959. In June 1959, an attack of vertigo and vomiting was accompanied by severe bifrontal headache and over 48 hours the patient became drowsy and mentally confused. When he was admitted to hospital, in addition to the drowsiness and mental confusion, the patient was found to be mute. In addition, on formal examination of the central nervous system, there was well marked nystagmus on lateral deviation of the eyes, a left sided Horner's syndrome, and a left facial palsy. There was also evidence of a marked right hemiplegia with sensory loss over the right side of the body. The degree of sensory loss could not be determined in view of the patient's mental state. Unfortunately, in view of the patient's mental confusion, the state of the visual fields could not be assessed. The patient remained in this state with minor fluctuations of consciousness for two and a half months before he died.

At post mortem the essential pathological findings were confined to the cerebral vessels and to the brain. On examination of the brain there was a bilateral infarction in the distribution of the calcarine fissure, but no occlusion of the posterior cerebral vessels could be demonstrated. In the cerebellum there was bilateral infarction of the territory of the superior cerebellar arteries and again no occlusion of these arteries could be found. Both the infarct of the cerebellum and the occipital lobes was considered to be of about between two and three months' duration. On examination of the vascular tree there was some minimal atheroma in the carotid arteries at their origin. Both posterior communicating arteries were extremely small, and this congenital anomaly was probably important in the pathogenesis of the infarction in this particular case. The examination of the vertebral arteries in the vertebral canal showed that there was a considerable difference in size between the two, the right being the larger. The left vertebral artery was partially occluded by atheroma at the level of the third cervical vertebra, but the right vertebral artery at the same level was completely occluded by both atheroma and thrombosis.

The findings in this patient are in every way typical of the syndrome of vertebral arterial occlusion.

The similarity between these patients and occlusion of the basilar artery has already been stressed, but there is one set of circumstances in which the differentiation between the two may be made, and that is where there is clinical evidence of disease of the major vessels of the aortic arch. Signs of disease of the major vessels of the aortic 
arch in the older age groups are due to atheroma in the majority of patients (Crawford et al., I959). It is interesting to recall that Broadbent in 1875 in a paper entitled 'Absence of Pulsation in both Radial Arteries, the Vessels being full of Blood,' described a patient with absent radial pulses due to atheroma of the subclavian arteries probably superimposed upon a congenital lesion. The vertebral artery in the patient he described was shown at post mortem to take an anomalous origin from the aortic arch rather than its usual site at the origin of the subclavian artery, and by doing so it avoided the effects of atheromatous disease at this site. Arterial occlusion and stenosis of the subclavian artery may frequently be present without symptoms, and in six patients recently observed with this condition only three complained of symptoms of ischaemia in the upper limbs. Four of these patients developed an intracerebral lesion and in three of these the intracerebral lesion had all the characteristics of a brain stem lesion. The following patient who came to post mortem is an example of this:-

\section{Case 4}

The patient, a man of 57 years of age, complained in 1957 of attacks of tingling in the left hand and a constant feeling of coldness and numbness of this limb. It was noted at the time that the blood pressure in the left arm was very much less than that in the right arm, and the pulses in the subclavian and axillary artery on the left were very much feebler than the right. The diagnosis of subclavian arterial stenosis was made at the time. He remained well for the next two and a half years, apart from an occasional complaint of tingling in the left hand, particularly in cold weather. In the spring of 1959 he complained of attacks of vertigo which came on quite suddenly, and when present caused some ataxia in walking. In August, 1959, he suddenly complained of an attack of weakness of the left arm, a weakness which recurred several times a day for a further seven days. On the third day following the first of these attacks of weakness of the arm he complained of sudden weakness of the left leg which lasted for 24 hours. On August 20, he developed persisting weakness of the left hand, and he was then admitted to hospital. On admission to hospital the blood pressure in the right arm was 145/95, but the left radial and brachial pulses were totally absent. Pulsation in the left subclavian artery could be felt but was diminished; the carotid pulses were of equal volume to palpation on both sides. Examination of the central nervous system at that time revealed a left lower facial palsy, some increased tone in the left arm, together with impairment of response to pin prick of the left hand, and probably also in the left leg.气̊ Position sense in the left fingers was also defective. $\bar{z}$ Both plantar responses were extensor. The $e_{\alpha}^{\mathbb{\Phi}}$ reflexes in the left arm were considerably briskerc. than in the right. The diagnosis of vertebral arterial occlusion at the level of its origin from the subclavian artery was made, and the patient made ${ }^{\circ}$ a slow recovery. Two months after this episode he was seized with sudden abdominal pain and? admitted to hospital where he died suddenly 120 hours after admission. At autopsy the cause of death was shown to be a mesenteric artery oc- $\vec{\circ}$ clusion. The vessels at the base of the brain:showed a moderate degree of atheroma and both $\vec{\omega}$ posterior communicating arteries were extremelyo small. The left vertebral artery was occluded by음 a plaque of atheroma at a point $1.5 \mathrm{cms}$. distal to its. origin from an atheromatous and narrowed sub- $\omega$ clavian artery. There was an old area of softening $\rightarrow$ on the left side of the brain stem. The carotid. and intracranial arteries were normal.

This combination of arterial insufficiency in anupper limb, coupled with a brain stem lesion, makes a distinctive clinical picture and important? to recognize, since it is clear that surgical treatment光 of atheroma at the origin of the vertebral artery may be as effective as that of the surgical treatmeg of carotid arterial disease (Crawford et al., 195\%.

No mention has so far been made of one clinical sign which is of considerable importance these lesions-the cervical bruit. Rob (1959), in emphasizing the value of the sign, estimated that a bruit was present in over 25 per cent. of cases $\stackrel{\square}{\bar{D}}$ with stenosis of the carotid artery and sometimes $\Rightarrow$ accompanied by a palpable thrill. The bruit is 3 often harsh in quality, systolic in timing, and conducted into the skull, with a maximal intensityọ. directly over the site of occlusion. When present such a bruit may be heard clearly over the orbit. 3 . A bruit in the supraclavicular fossa of similaro quality may be present with subclavian arterial disease.

\section{Radiology}

Routine radiology of the skull has little positive information of value to contribute to the diagnosis of extra- or intracerebral vascular disease. It is important, however, since by demonstrating pineal shift or changes in the bony contours of the $N$ skull an indication of other intracranial pathology may be given. Cerebral angiography, a technique which made possible the demonstration ofo thrombosis of the carotid artery in life, still remains $\widetilde{\varnothing}$ the most important single investigation. Not $\stackrel{\oplus}{+}$ only has it added certainty to the diagnosis of $\underline{T}$ occlusion of the major vessels, but it has provided $\stackrel{\vec{D}}{\vec{D}}$ a rapid means of differentiating these lesions from 
other intracranial lesions. The increasing use of angiography in vascular disease has emphasized the frequent difficulties of differentiating with any certainty on clinical grounds between intracranial and extracranial vascular occlusion.

Routine percutaneous carotid angiography will adequately demonstrate atheromatous disease at the origin of the internal carotid artery and occlusion of the larger arteries within the skull. Disease of the vertebral artery is more difficult to demonstrate because of the intrinsic difficulties in obtaining angiograms of the artery itself. It is generally agreed that direct puncture of the vertebral arteries when atheroma is suspected is undesirable, in view of the risk of precipitating thrombosis. Catheterisation of the brachial artery is satisfactory in expert hands (Sutton, 1959; Pygott and Hutton, 1959) or the alternative method of injecting through the subclavian artery in the supraclavicular fossa (Crawford et al., 1959; Morris, 1959). Pneumothorax occurs in ro to 20 per cent. of patients with the latter procedure, but it may be that this risk is preferable to direct puncture of the artery with its attendant danger of precipitating vascular occlusion. The most difficult technical problem is the demonstration of stenosis or occlusion of the origin of the major vessels of the aortic arch. Crawford et al. (1959) have used the method of Robb and Steinberg (1939) for visualizing the origin of these vessels, and they report that they have used this method satisfactorily on 2,0 patients for a variety of lesions without mortality or significant complications.

\section{Treatment}

At the present time a patient cannot be offered dietetic or other measures which will prevent with certainty the development of atheroma. Therefore, in the foreseeable future, treatment of extracranial vascular disease will be concerned with medical or surgical treatment of these lesions.

Medical treatment must include the management of the patient with the ' acute stroke,' and the recovering hemiplegia. Little need be said about the well-tried general principles of the treatment of such patients, but there is no doubt that success, in terms of recovery of function of the affected limbs, will in part be determined by the standard of efficiency of the nursing and physio-therapy which the patient receives in the acute stages. The other major problem in the treatment of these patients is the question of anti-coagulant therapy. Earlier reports on the treatment of cerebral thrombosis and embolism with anti-coagulant therapy (Foley and Wright, 1950) were hopeful, and the later observations of Millikan, Siekert and Shick (1955), Carter (1957) and Fisher (1958) encouraged their continued use. Millikan and his colleagues were particularly impressed with the value of anti-coagulant therapy in the treatment of the basilar artery insufficiency. Clearly there are two aspects to the problem which must be considered separately - the use of anti-coagulants in the acute phase and the use of long term anticoagulant therapy to prevent further occlusions in the cerebral vessels.

In considering the use of anti-coagulants in the acute phase of cerebral vascular disease there are two major difficulties. It has already been emphasized that the increasing use of angiography has underlined the difficulty, not only in differentiating between intra- and extra-cerebral vascular occlusion, but also in differentiating between cerebral thrombosis and a circumscribed cerebral haemorrhage. This clinical differentiation is essential in the safe use of anti-coagulants in the patient suffering from an acute cerebral vascular lesion. McKissock, Richardson and Walsh (1959) have recently reported an extensive study of spontaneous intracerebral haemorrhage, and illustrates in doing so the clinical difficulties encountered. The other problem in the acute phase is the theoretical possibility, which has some experimental support, that infarcts may become haemorrhagic with the use of anti-coagulants (Woods et al., 1958; Sibley et al., 1958).

The whole problem of anti-coagulants in cerebral vascular disease has recently been reviewed by Marshall and Shaw (1959). They emphasize that an estimate of the use of long term anticoagulants presents difficulties since so little is known of the natural history of the various types of cerebral vascular disease. They report their interim results of a controlled trial of anticoagulants in an acute cerebral vascular lesion and also the long term results of such treatment. It is to be hoped that this and other planned trials will finally answer the difficult but important question on the correct place of anti-coagulants in the treatment of cerebral vascular disease.

The surgical treatment of extracranial vascular disease is on firmer ground. Originally the surgical treatment was confined to arterectomy (Chao et al., 1938) or to sympathectomy (Johnson and Walker, 195I), but the results obtained by these methods were not very different from what one might reasonably anticipate in the natural history of the disease. The first successful arterial reconstruction was reported by Eastcott, Pickering and Rob (1954), and Rob (1959) has reviewed his later experience with 70 cases. His statement that 'good results usually follow surgery when the occlusion is partial and poor results when it is complete' summarizes his views. This clear cut difference between the results of surgery in partial and complete occlusions em- 
phasizes the importance of early diagnosis in the patient with extracranial vascular disease. $\mathrm{Al}$ though the surgery of complete occlusion of the carotid artery has been disappointing Rob makes the important point that operation on complete occlusions, if carried out early as an emergency before the thrombus has time to become adherent to the vessel wall, may very well be attended by greater success. Surgery of the vertebral artery has not advanced to the same degree as that of the carotid, but it is to be hoped that with improved radiological techniques reconstruction of the vertebral artery at its origin may also be possible as described by Crawford et al. (1958).

\section{Summary}

I. The pathology of extracranial vascular disease, as it affects the clinical picture of cerebral ischaemia, is described.

2. The clinical syndromes resulting from extracranial carotid and vertebral arterial disease are described.

3. The place of anti-coagulants in the treatment of cerebral vascular disease is discussed and the surgical treatment of carotid occlusion and stenosis is briefly reviewed.

\section{Acknowiedgments}

We wish to thank Professor D. A. K. Black and Mr. R. T. Johnson for permission to publish the clinical details of cases 2 and 3 respectively.

\section{REFERENCES}

ARING, C. D., (1945), Brain, 68, 28.

BEHRMAN, S. (1954), Postgrad. med. F., 30, 570.

BROADBENT, W. H. (1875), Trans. clin. Soc. Lond., 2, 165.
CARTER, A. B. (1957), Quiart. F. Med., 26, 335.

CHAO, W. H., KWAN, S. T. LYMAN, R. S., and LOUCKS, H. H. (1938), Arch. Surg. (Chirago), 37, 100.

CLARKE, E., and HARRIS, P. (1958), Lancet; ii, 1085. -

CRAWFORD, E..S., DE BAKERY, M. E., FIELDS, W. S., COOLEY, D. A., and MORRIS, G. C.'(1959), Circulation; 20, 168 .

CRAWFORD, E. S., DE BAKERY, M. E., and FIELDS, W. S. (1958), F. Amer. med. Ass., 168, 509.

DUFFY, P. E., and GRANT; G. B. (1958), Neurology, 8, 862.

EASTCOTT, H. H. G., PICKERING, G. W., and ROB, C. G. (1954), Lancet, ii, 994.

FISHER, C. M. (1958), Neurology, 8, 311 .

FISHER, C. M. (1954), Arch. Neurol. Psychiat. (Chicago), 72, 187. FOLEY, W. T., and WRIGHT, I. S. (1950), Med. Clin. N. Amer., 34, 909.

HICKS, S. P., and WARREN, S. (1951), Arch. Path., 52, 403. @

HOYT, W. F. (1959), A.M.A. Arch. Ophth., 62, 260.

HUTCHINSON, E. C., and YATES, P. O. (1957), Lancet, $1,2$.

JOHNSON, H. C., and WALKER, A. E. (195I), f. Neurosurg.,

KALMANSOHN, R. B., and KALMANSOHN, R. W. (1957), Circulation, $15,237$.

KUBIK, C. S., and ADAMS, R. D. (1946), Brain, 69, 73.

MCKISSOCK, W., RICHARDSON, A., and WALSH, L. (1959), 3 Lancet, ii, 683 .

MARSHALL, J., and SHAW, D. A. (1959), Proc. roy. Soc. Med.,

52, 547. Clin., 30, 93 .

MILLIKAN, C. H., SIEKERT, R. G., and SHICK, R. M. (1955), $\vec{A}$ Ibid., 30, 116.

MORRIS, L. (1959), Brit. F. Radiol., 32, 673

PICKERING, G. W. (1959), Proc. roy. Soc. Med., 52, 540.

PYGGOTT, F., and HUTTON, C. F. (1959), Brit. F. Radiol., $32,114$.

ROB, C. G. (1959), Proc. roy. Soc. Med., 52, 549. ROBB, C. P., and STEINBURG, I. (1939), Amer. F. Roentgenol., $\frac{\mathrm{C}}{2}$
41, I.

ROSEGAY, H., and WELCH, C. K. (1954), Ұ. Neurosurg., II, 363.

SIBLEY, W. A., MORLEDGE, J. H., and LAPHAM, L. Z. (19,8), Amer. F. med. Sci., 234, 663.

SYMONDS, C., and MACKENZIE, I. (1957), Brain, 80, 415-

SUTTON, D. (1959), Brit. F. Radiol., 32, 283.

STOPFORD, J. S. B. (1921), f. Anat. (Lond.), 50, 131.

VAN DER EECKEN, H. M., and ADAMS, R. D. (1953), F. Neuropath., 12, 132 .

WEISS, S. (1938), Res. Publ. Ass. nerv. ment. Dis., 18, 571.

WOOD, M. W., WAKIM, K. G., SAYRE, G. P., MILLIKAN, 응 C. H., and WISHNANT, J. P. (1958), Arch. Neurol. Psychiat. (Chicago), 79, 390.

YATES, P. O. (1954), Proc. roy. Soc. Med., 7, 606.

\section{RUTHIN CASTLE, NORTH WALES}

A Clinic for the diagnosis and treatment of Internal Diseases (except Mental or Infectious Diseases). The Clinic is provided with a staff of doctors, nurses, technicians, modern Radiological and Physiotherapy departments.

The surroundings are beautiful. The climate is mild. There.is central heatinz throughout. The annual rainfall is 30.5 inches, that is less than the average for England.

The Fees are inclusive and vary according to the room occu oied.

For particulars apply to THE SECRETARY, Ruthin Castle, North Wales. 